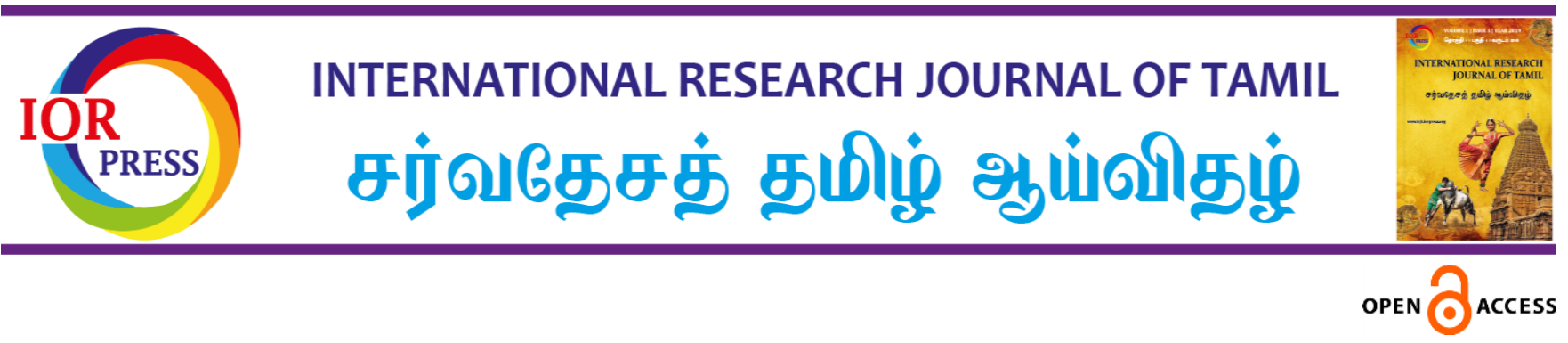

\title{
சிற்றிலயக்கியங்களில் பள்ளிசை
}

\section{லூ. ஜனார்த்தனன் அ,*}

அ இசைத்துறை, அண்ணாமலை பல்கலைக்கழகம், சிதம்பரம்-608002, தமிழ்நாடு, இந்தியா

\section{Sitrilakiyangalil Pallisai}

\author{
L. Janarthanan a, * \\ a Department of Music, Annamalai University, Chidambaram-608002, Tamil Nadu, India
}

* Corresponding Author: janaartist79@gmail.com

Received: 04-11-2020

Revised: 13-06-2021

Accepted: 18-06-2021

Published: 23-06-2021

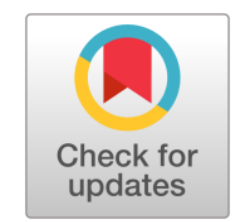

\begin{abstract}
It is cynical to set out to give any one of the four affirmative meanings of virtue, meaning, pleasure, and home. In this way, Pallu, Kuravanchi, Nondi, Kuluvam, Makudi etc. are found to have artistic qualities in them. Pillai Tamil, Kalambakam, Satakam, Malai and Anthadi are found to be literary. The action of the tooth has acquired a pronoun and has become called a tooth. Those who work in a place full of potholes are referred to as Pallar. Although Pallu literature later took literary form, its elements can be traced back to ancient literature. Various elements must have been supplemented in order to get the full text of the school literature. Such literary genres are written with a tendency to explain a variety of meanings. Yet they are all suppressed together in the sense that they come together in giving hints about music. The literary genre of cognition, one of the eight categories referred to by the tholkappiyam, applies to 'pallu vagai' literature. The biological condition of the pallu, in its entirety and in its simplest form, is made clear to us in the form of short stories and songs. In this article you will find what the Psalms say about agriculture, the God of the pallar, their family, way of life and music.
\end{abstract}

Keywords: Sitrilakiyangam, Pallu, Kuravanchi, Nondi, Kuluvam, Makudi

முன்னுரை

அறம், பொருள், இன்பம், வீடு எனும் நான்கு உறுதிப் பொருள்களுள் ஏதேனும் ஒன்றைத் தருவதாக அமைவது சிற்றிலக்கியம். இவ்வகையில் பள்ளு, குறவஞ்சி, நொண்டி, குளுவம், மகுடி போன்றவை அவற்றுள் அடங்கியுள்ள கலைத் தன்மை கொண்டவையாக காணப்படுகின்றன. பிள்ளைத்தமிழ், கலம்பகம், சதகம், மாலை, அந்தாதி என்பன இலக்கியத்தன்மை கொண்டவையாக காணப்படுகின்றன.

பள் என்ற செயல் உகர விகுதி பெற்று பள்ளு என்று ஆகிஉள்ளது. பள்ளங்கள் நிறைந்த இடத்தில் வேலை செய்வோரைப் பள்ளர் என்று குறிப்பிட்டனர் பள்ளர்கள் பாடும் பாடலே பள்ளு என்று கூறுவார். பள்ளு இலக்கியம் பிற்காலத்தில் இலக்கிய வடிவம் பெற்றாலும் அதன் கூறுகள் பண்டைய இலக்கியங்களிலேயே தென்படுகின்றன. பள்ளு இலக்கியம் முழுவடிவைப் பெறுவதற்கு பல்வேறு கூறுகள் துணை செய்திருக்க வேண்டும். 
இத்தகைய இ இலக்கிய இ இனங்கள் பலவித பொருள்களை விளக்கும் போக்குடன் எழுதப்பட்டுள்ளன. ஆனாலும் இசை பற்றிய குறிப்புக்களைத் தருவதில் ஒன்றுபடுவன என்ற எண்ணத்தில் அவை அனைத்தையும் ஒன்றாக அடக்கிச் சிந்திக்கப்படுகிறது.

தொல்காப்பிய நூலில்

‘சேரி மொழியாற் செவ்விதிற் கிளந்து

தேர்தல் வேண்டாது குறித்தது தோன்றிற்

புலனென மொழிப் புலனுணர்ந் தோரே'

என்று பள்ளைக் குறித்து தொல்காப்பியத்தில் பாடலாக குறிப்பிடப்பட்டுள்ளது. தொல்காப்பியர் குறிப்பிடும் எட்டு வகைப்பிரிவில் ஒன்றான புலன் என்னும் இலக்கிய வகை 'பள்ளு வகை' இலக்கியத்திற்குப் பொருந்தும் என்பர். இசையைக் குறித்து பள்ளுப் பாடல்கள் என்ன கூறுகிறது என்பதை இக்கட்டுரையில் காணலாம்.

\section{பள்ளிசையின் நோக்கம்}

உழவர் வாழ்வை சித்தரிக்கும் இலக்கியமானது பள்ளு. மருத நில நூலாகக் கருதப்படுவது பள்ளு. சீரி மொழியும் செந்தமிழ் வழக்கும் கலந்த நூல் பள்ளு. ஏசல் இடம்பெறும் இலக்கியம் பள்ளாகும். சமயக் கருத்துக்களையும், வாழ்வியல் முறைகளையும் சுவையாகக் கூறியுள்ளது. பள் இலக்கியத்திற்கு உழத்திப் பாட்டு என்ற வேறுப் பெயரும் உண்டு. சந்த இசை மிகுந்து காணப்படும். வு.மு. சிதம்பரம் அவர்கள் பள்ளுப் பாடல்களை கோலாட்டாமாக பாடியுள்ளார் என்று தனது நூலில் கூறியுள்ளார். இவரே முக்கூடர்ப் பள்ளு, முதற்பள்ளு என்று கூறியுள்ளார். கமலேயின் ஞானபிரகாசம் எழுதிய திருவாரூர் பள்ளு, முதற்பள்ளு என்பார் ந.வி.ஜெயராமன் அவர்கள். வைணவமும், சைவமும் கலந்த ஓர் அழகான இலக்கிய நூல் பள்ளாகும். 150 நெல் வகைகளையும் 120 மீன் வகைகளையும் குறித்து பள்ளில் கூறப்பட்டுள்ளது. சின்னதம்பி வேளாளர் அவர்கள் முக்கூடர் பள்ளுவை, முக்கூடல் நாடகமாக்கினார். பேதுரு புலவர் அவர்கள் ஞானப்பள்ளு என்ற கிறிஸ்த்தவ சமயம் சார்ந்த பள்ளை இயற்றியுள்ளார்.

பள்ளர்களின் சமயம் வாழ்வியல் அவர்கள் உணவான நெல், மீன் இவைகளை முழுமையாக எடுத்துக் கூறிய சிற்றிலக்கியங்களில் ஒன்றான பள்ளில், இசை, நாடகம் ஒன்றாக கலந்து இருக்கும். பள்ளில் இசைக் குறித்து இக்கட்டுரையில் காணலாம்.

\section{பள்ளில் இசை}

நாட்டுப்புற இலக்கிய வகைகளில் ஏசல் வகையைச் சேர்ந்தது பள்ளு. பள்ளு என்பது உழவுச் செய்கின்ற நிலங்களைக் குறித்துப் பாடப்படுவதாகும். பள்ளு என்பது இரண்டு, மூன்று நூற்றாண்டுகளாகத் தமிழ்நாட்டில் மிக பரவலாகப் பழக்கத்திலிருந்த உழவர்களின் இசை நாடகம். முழுமையும் இது இசைப் பாடல்களினால் ஆக்கப்பட்டு உள்ளது. நிகழ்ச்சிகள் பாடல்களால் விளக்கப்படும். பாடல்கள் மக்கள் முன்பு பாடப்பட்டு அவற்றுக்குத்தக்கவாறு நடித்துக் காட்டப்படும். இசையின்றி இத்தகைய நாடகங்களுக்குச் சுவை இல்லை. வாழ்வில் நடக்கும் பல நிகழ்ச்சிகளை ஒரு விளக்கக் காட்சிக்குள் அடக்கி இசைப்பாடல்களால் அவற்றை வெளியிடுவதில் பள்ளு போன்ற இசைமலிந்த நாடகங்கள் மக்களின் ஆதரவைப் பெறுகின்றன. இவற்றில் இசைக்குப் பெரும்பங்கு உண்டு. இசையின்றி பள்ளு போன்ற நாடகங்கள் இல்லை என்று கூறலாம். பள்ளின் இசைத் தன்மைகளைக் காண முயலலாம் (Perumal, 1984).

தமிழ்நாட்டில் நூற்றுக்கணக்கான பள்ளுகள் நடிக்கப் பெற்றுள்ளன. அவற்றுள் ஒருசில நூல்வடிவம் பெற்றன. நூலாகக் கிடைத்துள்ள பள்ளுகளில் சிறந்தது முக்கூடற் பள்ளு. அவை அடிப்படையாகக் கொண்டு பள்ளின் இசையமைப்பு பற்றி அறியலாம். 


\section{பள்ளின் இசைத்தன்மை} காட்டும்.

முக்கூடற் பள்ளு இசைத் தன்மையுடையது என்பதை அதனுயை காப்புச் செய்யுளே நன்கு

'பூமேவு நீலவெற்பிற் பொன்னிறமு முண்டெனப்பூ

மாமேவு முக்கூடல் மாலழகர் பள்ளிசைக்கே

பாமேவு சொற்புரப்பார் பாவலரென் றெட்டெழுத்து

நாமேவு பத்தர்பத்து நாவலரும் காப்பாமே'.

பள்ளிசைக்கே’ என்று குறிப்பிடுவதில் இது ஓர் இசைப்பாடல் நூல் என்பது மிகத்தெளிவு.

இசைக் குறிப்புகாட்டிப் பாடல்களைத் தொடங்கும் போக்கு இன்னும் தெளிவாகப் பாடல்களின் இசைத்தன்மையைக் காட்டும். முதலில் திருமால் வணக்கப் பாடலைக் காணலாம் (Puliyur Kesikan, 2020).

‘தன தன தனத் தானன் தானன தானத் தனனா'

இது வரவிருக்கும் பாடலை எவ்வாறு இசையமைத்துப் பாடவேண்டும் என்பதற்கான மெட்டமைப்பு. அடுத்துப் பாடல் தரப்படுகிறது.

'மணி மரகதச் சோதியர் ஆதியர்

மனு மரபினிற் காவலர் கோவலர்

மறை முதல்வர்முக் கூடலர் ஏடலர்- வாசத்துளவோர்' (மேற்படி: பா.1: 1)

இவ்வாறு இந்தப் பாடலில் நாலு அடிகள் உள்ளன. ஒவ்வொரு அடியும் மூன்று மடக்காக அமைகிறது. இறுதியில் ஒரு தனி மடக்குக் குறுகியதாக உள்ளது (Puliyur Kesikan, 2020). மூன்று மடக்குகளுக்கும் ஒரே மாதிரியான இசையில் மெட்டை அமைத்து தனி மடக்குக்குத் தனியாக மெட்டை குறிப்பிட்டுக் காட்டப்படுவதைக் காணலாம். நான்கு அடிகளையும் இவ்வாறு மாறி மாறிப் பாடவேண்டும். இசை அமைந்து விடும்.

இம்முறையைத் தெளிவாகக் காணவேண்டுமானால் கருடாழ்வார் பாடலுக்கு மெட்டமைத்துக் காட்டியுள்ளதைப் பார்த்து நன்கு அறியலாம்.

மெட்டு

$$
\begin{aligned}
& \text { தானத்தன தானத் தனதத் } \\
& \text { தானத்தன தானத் தனதத் } \\
& \text { தானத்தன தானத் தனதத்- } \\
& \text { தனன தனத்தா }
\end{aligned}
$$

பாடல்

$$
\begin{aligned}
& \text { தாரத்திரு பேரிற் தனதத் } \\
& \text { தாகத்தின ளாகத் தருமச் } \\
& \text { சாலைப்புவி காலைத் தடவ- } \\
& \text { தலையணை கொடுத்தே. }
\end{aligned}
$$

மெட்டையும் பாடலையும் பொருத்திக் காணலாம். மூன்று மடக்கும் ஒரே மாதிரி இசையில்அமையத் தனிமடக்கு வேறு மெட்டில் இருப்பதைப் பார்க்கலாம். இசையமைப்பு இவ்வாறு அமையினும் இவற்றுள் அமையும் பண் ஒன்றாகவே இருக்கும். பாடலுக்குத் தக்கவாறு மெட்டமைப்பு 
என்பதைச் சொல்லமைப்பு கொண்டு பார்க்கலாம். மூன்று மடக்குகளிலும் முதற்சீர் நீண்டு இடைச்சீர் குறுகி மூன்றாம் சீர் இடைப்பட்டு அமைவதைக் கண்டுணரலாம். ஆனால் தனிமடக்கில் இரண்டு சீர்களும் நீண்டு உள்ளன. அவை அமைப்பில் வேறுபாடு கொண்டுள்ளன. ஆகையினால் மெட்டமைப்பில் மாற்றம் காணப்படுகிறது (Puliyur Kesikan, 2020). இசைபற்றி நன்கு தெரிவதுடன் இசைத்துப் பாடவும் தெரிந்தால் தான் இசைப்பாடல்களை எளிதாக அமைத்தெழுத முடியும். அக்காலப் புலவர்கள் இலக்கிய இலக்கண இசைப் புலமை கொண்டவர்களாக இருந்தமையினால் இவ்வாறான பாடல்களை எளிதாகவும் எளிமையாகவும் எழுதியுள்ளனர்.

\section{ஓப்பாய்வு}

பள்ளிசைப் பாடல்கள் சிற்றிலக்கியங்களில் மிகவும் எளிமையான சந்தங்களைக் கொண்டு, நாட்டுப்புற கலையான கோலாட்டத்தில் பாடி ஆடி வருகின்றனர். பள்ளு இலக்கியத்தின் முதல் நூலாக முக்கூடற்பள்ளு என்ற நூல் அமைகின்றது. இந்நூல் 17-ஆம் நூற்றாண்டின் இறுதியில் தோன்றியது என்பர். அதன் பின்னர் ஞானப்பள்ளு, திருவாரூர்ப் பள்ளு, குருகூர்ப் பள்ளு, சிவசயிலப் பள்ளு, வைசியப்பள்ளு, வடகரைப் பள்ளு, திருமலை முருகன் பள்ளு, சீகாழிப் பள்ளு, செண்பகராமன் பள்ளு, தில்லைப் பள்ளு, வையாபுரிப் பள்ளு, கண்ணுடை அம்மை பள்ளு, திருப்புன வாயிற் பள்ளு, கதிரை மலைப் பள்ளு, பறாளை விநாயகர் பள்ளு, தண்டிகைக் கனகராயன் பள்ளு, குற்றாலப் பள்ளு, திருச்செந்தில் பள்ளு, போரூர்ப் பள்ளு, இருப்புலிப் பள்ளு, திருவிடை மருதூர்ப் பள்ளு, புதுவைப் பள்ளு போன்ற பல நூல்கள் தோன்றின (Sundram, 1994).

தமிழர் வாழ்வு பாட்டோடு இரண்டறக் கலந்ததாகும். பிறந்தவுடன் தாலாட்டிலிருந்து தொடங்கும் பாட்டு இறக்கும்போது ஒப்பாரியில் முடிகிறது. இவை தவிர அதிகமாகப் பாடுபவர்கள் தொழிலாளர்கள். பாட்டில் அவர்கள் தங்கள் உழைப்பின் வலியை மறக்கிறார்கள். வண்டி ஓட்டும் போது, ஏற்றம் இறைக்கும்போது, களை எடுக்கும் போது, நாற்று நடும்போது, அறுவடையின் போது உழவர்கள் பாடுவது வழக்கம். இது உழத்திப பாட்டு என்று வழங்கப் பட்டதை பன்னிரு பாட்டியல் நூலிலிருந்து சான்றுடன் குமார.பத்மநாபன் விளக்குகிறார்.

\section{முடிவுரை}

பள்ளு என்ற இலக்கிய வகைக்குப் பள்ளு நாடகம், பள்ளு மூவகைத்தமிழ், பள்ளேசல், பள்ளிசை என்ற பெயர்களும் காணப்படுகின்றன. இப்பெயர்களால் பள்ளு என்ற இலக்கிய வகை நடித்தற்கு உரியது. பாடுவதற்கு உரியது. இயல், இசை, நாடகம் என்ற முத்தமிழுக்கும் உரியது என்பன தெரிய வருகின்றன. சிற்றிலக்கியங்களிலேயே பள்ளும், குறவஞ்சியும் தான் வாசிக்க இலகுவானவை. மேலும் இதுவரை 35 பள்ளி இலக்கியப் பெயர்கள் கண்டறியப்பட்டுள்ளன. அவற்றில் எத்தனை பதிப்பிக்கப்பட்டன என்பது தெரியவில்லை என்று தம் சிற்றிலக்கியங்கள் நூலில் நாஞ்சில் நாடன் கூறுகிறார். பள்ளிசை அனைத்து சமயங்களிலும் பாடப்பட்டது. இஸ்லாமியர் சமயத்தில் மட்டும் எந்த பள்ளு இலக்கிய நூலூம் உருவாகவில்லை.

\section{References}

Perumal A.N., (1984) Tamilisai, International Institute of Tamil Studies, Chennai, India.

Puliyur Kesikan, (2020) Mukkoodar Pallu Moolamum Uraiyum, Gowra Puthaga Maiyam, Trichy, India.

Sundram V.P.K., (1994) Tamilisai Kalaikalanjiyam, Bharathidasan University, Tiruchirappalli, India.

Funding: NIL

Acknowledgement: NIL 


\section{Conflict of Interest: NIL}

About the License:

\section{(C)(1)}

Attribution 4.0 International (CC BY 4.0)
(C) The author 2021. The text of this article is licensed under a Creative Commons Attribution 4.0 International License 